\section{"Naciones unidas para la salud mental"}

La Organización Mundial de la Salud ha establecido un programa especial para promover la salud mental en poblaciones necesitadas de servicios. Este programa, designado "Naciones unidas para la salud mental", está dirigido a mejorar la salud mental y el bienestar psicosocial de la población mundial, con énfasis en aquellas poblaciones que han tenido solo un acceso limitado a los servicios de salud mental.

Iniciado y coordinado por la OMS, "Naciones unidas para la salud mental" ha sido configurado como un programa especial dentro del sistema de las Naciones Unidas. Tal como indicara el Secretario General de esa organización, el objetivo del sistema en general es "promover la salud mental y el bienestar de todos los habitantes del planeta". El nuevo programa hará hincapié en las áreas siguientes:

- Planes nacionales sobre salud mental

- Mejora de los servicios y tratamientos

- Derechos humanos y legislación

- Facilitación de iniciativas por consumidores y familias

- Promoción de la salud mental y prevención de trastornos mentales, prestando atención especial a las mujeres, los niños y los adolescentes

- Programas especiales de salud mental que beneficien a refugiados y poblaciones indígenas.

Uno de los objetivos del programa es atraer la atención del público en general y de los gobiernos hacia los efectos que tienen los problemas sobre la salud mental y el abuso de sustancias en la salud física y el bienestar social de las poblaciones necesitadas. El primer paso en esa dirección es llevar a cabo una serie de acontecimientos prestigiosos para dar a conocer al público la importancia de la salud mental. Se estimulará la participación de figuras políticas importantes procurando centrar los esfuerzos en conseguir el compromiso político de las autoridades encargadas de tomar decisiones. El programa especial se propone crear un proceso internacional de concienciación y cimentar la voluntad y el compromiso políticos para que las dificultades de salud mental se perciban como un problema de salud pública importante y se haga frente al estigma y la discriminación que se asocian con ellas.

Otro objetivo es definir y promover las estrategias de colaboración para mejorar la salud mental 
que puedan ponerse en marcha en forma de proyectos nacionales de cooperación técnica coordinados por organismos del sistema de las Naciones Unidas con la cooperación de otras organizaciones gubernamentales y no gubernamentales. Se hará énfasis sobre todo en los proyectos que favorezcan a niños en circunstancias difíciles, mujeres maltratadas, personas traumatizadas por la violencia, personas adictas al alcohol o a las drogas, personas con enfermedades mentales crónicas y las que viven en pobreza extrema.

El programa establecerá varios proyectos modelo en cada una de las seis regiones de la OMS. Mediante esos proyectos se espera demostrar los esfuerzos de colaboración que pueden lograrse en los propios países y establecer el prototipo para proyectos de mayor envergadura. Ya se han iniciado una serie de esos proyectos en la Argentina, Sri Lanka, Vietnam y Yemen, algunos para poblaciones indígenas del Brasil, Canadá y algunas islas del Pacífico, y hay varios otros en consideración.

El común de la gente, los encargados de tomar decisiones en el sector de la salud y la mayoría de los profesionales de la comunidad médica no están al tanto del carácter y el alcance de los trastornos mentales y del precio social y económico que tienen que pagar tanto los pacientes que sufren como sus familias y sus países. Si bien los problemas mentales y del comportamiento no figuran como causas principales de muerte, se encuentran entre las 10 causas más importantes de discapacidad duradera, se presentan con frecuencia durante los años más productivos y dejan huellas que duran toda la vida.

Como los efectos discapacitantes de las enfermedades mentales imponen una pesada carga en la sociedad y la economía, la ejecución de este programa mundial requiere movilizar fuerzas sociales, económicas y políticas a la vez, y realizar cambios sustantivos en las políticas gubernamentales relacionadas con la educación, la salud y el desarrollo económico de cada país. Es evidente que un programa de esta magnitud necesita el empeño intenso y continuo de todas las naciones, con la cooperación recíproca de gobiernos, organizaciones no gubernamentales y organismos especializados del sistema de las Naciones Unidas. De las contribuciones voluntarias de gobiernos, fundaciones, individuos y otras fuentes depende que ese empeño se convierta en realidad.

Para información adicional sobre este nuevo programa, póngase en contacto con: Director, División de Salud Mental y Prevención del Abuso de Sustancias, Organización Mundial de la Salud, 20 Avenida Appia, 1211 Ginebra 27, Suiza (fax: +41 22 7914160; teléfono: +41 22 7913617).

\section{SYNOPSIS}

\section{Nations for Mental Health}

The World Health Organization has established a special program called Nations for Mental Health to promote mental health in underserved populations, with special emphasis on women, children, adolescents, refugees, and indigenous populations. One of the program's objectives is to stimulate greater public and governmental awareness of the social and economic cost of mental illness and substance abuse. A second objective is to identify and promote collaborative strategies for improving mental health that can be implemented through country-level technical cooperation projects by organizations of the United Nations system, in cooperation with other international governmental and nongovernmental organizations. A number of demonstration projects are already under way and others are planned. 